\title{
Higher Placental Anti-Inflammatory IL-10 Cytokine Expression in HIV-1 Infected Women Receiving Longer Zidovudine Prophylaxis Associated with Nevirapine
}

\author{
Sakorn Pornprasert ${ }^{1}$, Jean-Yves Mary ${ }^{2}$, Albert Faye ${ }^{3}$, Pranee Leechanachai ${ }^{1}$, Aram Limtrakul ${ }^{4}$, \\ Sungwal Rugpao ${ }^{5}$, Pannee Sirivatanapa ${ }^{5}$, Vorapin Gomuthbutra ${ }^{6}$, Wanmanee Matanasaravoot ${ }^{7}$, \\ Sophie Le Cœur ${ }^{8}$, Marc Lallemant ${ }^{9}$, Françoise Barré-Sinoussi ${ }^{10}$, Elisabeth Menu ${ }^{10}$ and \\ Nicole Ngo-Giang-Huong ${ }^{*, 9}$ for the ANRS 1267 study team and the HIV-1 PMTCT-PlaNet
}

\begin{abstract}
${ }^{1}$ Department of Medical Technology, Faculty of Associated Medical Sciences, Chiang Mai University, Chiang Mai, Thailand; ${ }^{2}$ INSERM U717, Université Paris 7, Paris, France; ${ }^{3}$ INSERM U131, Paris, France; ${ }^{4}$ Health Promotion Center Region 10, Chiang Mai, Thailand; ${ }^{5}$ Department of Obstetrics and Gynecology, Faculty of Medicine, Chiang Mai University, Chiang Mai, Thailand; ${ }^{6}$ Nakornping Hospital, Chiang Mai, Thailand; ${ }^{7}$ Lamphun Hospital, Lamphun, Thailand; ${ }^{8}$ Institut National d'Etudes Démographiques, Paris, France; ${ }^{9}$ Institut de Recherche pour le Développement, UMI 174/ PHPT, Chiang Mai, Thailand; ${ }^{10}$ Unité de Régulation des Infections Rétrovirales, Institut Pasteur, Paris, France
\end{abstract}

\begin{abstract}
Placental cytokine balance may be critical for the control of mother-to-child transmission (MTCT) of HIV. We assessed whether the type and duration of antiretrovirals used for prevention of HIV-1-MTCT modified the inflammatory cytokine profile. We investigated the levels of cytokine expression in the placentas of 61 HIV-1-infected women who received zidovudine (ZDV) plus single dose nevirapine (SD-NVP) or ZDV only for prevention of MTCT. Placentas of 38 HIV-1-uninfected women were included as controls. All placentas were obtained after vaginal delivery. Levels of mRNA and cytokine expression were quantified using real-time PCR and ELISA, respectively, in placental explants and 24-hour culture supernatants and analyzed in relation to the women's characteristics and the type and duration of antiretroviral prophylaxis. HIV-1-infected and uninfected women did not show any differences in the expression of placental cytokine secretion except for a trend toward lower TNF- $\alpha$ mRNA levels in HIV-1-infected women. Within the HIV-1-infected group, women who were exposed to a long duration of ZDV ( $>72$ days) or received SD-NVP less than $5 \mathrm{~h}$ prior to delivery, more frequently expressed detectable levels of IL-10 in their placentas $(32 \%$ versus $7 \%(p=0.01)$ and $32 \%$ versus $5 \%(\mathrm{p}=0.02)$, respectively). No infant was found to be HIV-1-infected. Our results showed a normalization of the placental cytokine balance in HIV-1-infected women receiving antiretroviral prophylaxis. Furthermore, the type and duration of antiretroviral prophylaxis have an impact on the placental anti-inflammatory IL-10 expression level, which may contribute to controlling HIV replication at the placental level, thus reducing MTCT of HIV-1.
\end{abstract}

Keywords: HIV-1, placenta, antiretroviral prophylaxis, zidovudine and nevirapine, IL-10 cytokine, mother-to-child transmission.

\section{INTRODUCTION}

The placenta constitutes a powerful natural barrier to HIV-1 transmission, presumably through its double layer of polarized cells, cytotrophoblasts and syncytiotrophoblasts. Indeed, primary cultures of purified human trophoblasts [1, $2]$ and trophoblast cell lines [3,4] display limited permissiveness for HIV-1 infection by cell-free viruses and restricted HIV-1 replication. Cytokine balance in the placental environment is known to play an important role not only in the maintenance of pregnancy but also in favoring or inhibiting in-utero infection. For example, elevated levels of placental tumor necrosis factor-alpha $(\mathrm{TNF}-\alpha)$ were associated with poor pregnancy outcome and higher risk of transmitting malaria to the infant [5-7]. HIV-1 can up-regulate placental interleukin (IL)-1 $\beta$, IL-6, and TNF- $\alpha$ pro-inflammatory

*Address correspondence to this author at the Institut de Recherche pour le Développement (IRD), UMI 174/PHPT, 29/7-8 Samlan Road, Soi 1, Prasing, Muang, Chiang Mai, 50200, Thailand; Tel: 6653894 431; Fax: 6653 894220; E-mail: nicole@phpt.org cytokines in vitro $[8,9]$. These pro-inflammatory cytokines have been shown to activate HIV-1 proviral expression in trophoblast cells [10-12].

Antiretroviral prophylaxis during pregnancy and labor, and in neonates dramatically reduces perinatal HIV-1 transmission. In the PACTG 076/ANRS 024 trial, the use of zidovudine (ZDV) prophylaxis in non-breastfeeding women during pregnancy and labor and in neonates lowered the risk of transmission by two-thirds [13]. A single dose of nevirapine (SD-NVP) given to women at the onset of labor and to infants 48-72 hours after birth decreased the risk of transmission by almost half [14]. When SD-NVP was administered to women at the onset of labor in addition to ZDV prophylaxis starting from 28 weeks of gestation, mother-to-child HIV-1 transmission was reduced to less than $2 \%$ [15]. The effect of these antiretroviral drugs on decreasing maternal HIV viral load is insufficient to fully explain their efficacy for the reduction of perinatal HIV-1 transmission rates. For example, in the PACTG 076/ANRS 024 trial, the median viral load decrease associated with ZDV prophylaxis in HIV-1 infected pregnant 
women was $0.3 \log _{10}$ copies/ml [16]. In the HIVNET006 study, a single dose of nevirapine given at the onset of labor resulted in a median viral load decrease of $1.3 \log _{10}$ copies $/ \mathrm{ml}$ at 1 week postpartum [17]. This suggests that there are additional mechanisms through which antiretrovirals prevent MTCT of HIV-1. Since both drugs easily cross the placenta, a pre- or post-exposure prophylactic effect in the neonate is very likely [15]. In addition, modification of the placental cytokine environment may contribute to reducing transmission.

Our objective was to determine whether the type and duration of antiretrovirals used for prevention of HIV-1 MTCT can modify the placental cytokine balance.

\section{MATERIALS AND METHODS}

\section{Study Population}

This prospective study was conducted in 4 hospitals in Chiang Mai and Lamphun provinces in northern Thailand. The protocol was approved by the Ethics Committee of the Faculty of Associated Medical Sciences, Chiang Mai University. All women participating in this study provided written informed consent. Women who had a vaginal delivery and did not give birth to twins were enrolled. HIV-1 infected women who delivered by caesarean section were excluded from the analysis.

Between April 2002 and September 2004, ninety-nine placentas, 61 from HIV-1 infected women and 38 from HIV1 uninfected women, were collected. Fifty-nine of the HIV-1 infected women, enrolled at Health Promotion Center Region 10, Nakornping, and Lamphun hospitals, were participating in PHPT-2 [15] (ClinicalTrials.gov NCT00398684). They all received ZDV (300 mg twice daily) starting at 28 weeks' gestation, or as soon as possible thereafter. According to their randomization arm, women received either a single dose of NVP $(200 \mathrm{mg})$ or a placebo at onset of labor. The remaining two HIV-1 infected women, enrolled at Maharaj Nakorn Chiang Mai hospital, received ZDV starting at 34 weeks' gestation.

The following data were collected from all women: age, gestational age at delivery, and mode of delivery. For HIV-1 infected women, the following additional data were collected: antiretroviral prophylaxis (type, timing, and duration), CD4 cell count (cell $/ \mathrm{mm}^{3}$ ) before 28 weeks of gestation and plasma HIV-1 RNA viral load at delivery $\left(\log _{10}\right.$ copies $/ \mathrm{ml}$ ).

Diagnosis of HIV-1 infection in infants born to HIV-1 infected mothers was performed using DNA PCR on dried blood spots (Amplicor ${ }^{\circledR}$ HIV-1 DNA assay version 1.5, Roche Molecular Systems Inc., Branchburg, NJ, USA) as previously described [15]. For infants born to women enrolled at the Maharaj Nakorn Chiang Mai hospital, HIV-1 infection was assessed by HIV-1 serology (HIV Combi, Elecsys $^{\circledR}$, Roche, Germany and Anti-HIV-1/2 Plus, Enzygnost $^{\circledR}$, Dade Behring, Germany) at 18 months of age.

\section{Placenta Processing}

Within three hours post-delivery, placentas were processed to isolate placental chorionic villi as previously described [18]. Fragments $(<0.5 \mathrm{~cm}$ of thickness) of placental chorionic villi were immersed in $1.5 \mathrm{~mL}$ of RNA stabiliza- tion reagent (RNAlater ${ }^{\mathrm{TM}}$, Qiagen, Germany), stored immediately at $2-8^{\circ} \mathrm{C}$ for 24 hours, and then transferred to $-70^{\circ} \mathrm{C}$. Six grams of placental chorionic villi were cultured for 24 hours in 2 flasks ( $3 \mathrm{~g} /$ flask), each containing $20 \mathrm{~mL}$ of culture medium as previously described [18].

\section{Quantification of Secreted Cytokines}

Quantification of TNF- $\alpha$, IL-8, IL-10, IL-15, IL-16, and regulated on activation normal $\mathrm{T}$-cell-expressed and secreted (RANTES) in the placental culture supernatants was performed using a quantitative sandwich enzyme immunoassay (ELISA) technique with cytokine specific commercial kits as previously described [18]. Quantification of leukemia inhibitory factor (LIF) was performed at the laboratory of Immunology, CNRS UMR 5540, Université Bordeaux 2, France, using an in-house sandwich ELISA [19].

\section{Quantification of Cytokine mRNA}

Total RNA was extracted from thawed placental chorionic villi and reverse transcribed to cDNA using the Taqman ${ }^{\circledR}$ Reverse transcription kit (Applied Biosystems, Foster City, CA, USA). Stromal cell-derived factor (SDF)-1, TNF- $\alpha$, IL-8 and IL-10 mRNA levels were quantified using the Taqman real-time PCR assay as previously described [18]. The cytokine mRNA levels are expressed as multiples of the results obtained with reference cDNA, prepared from a term placental tissue of an HIV-1 uninfected woman, used as a calibrator $(\times$ cal).

\section{Statistical Analysis}

Characteristics of the 2 groups, HIV-1 infected and uninfected women, are described by n (\%) for qualitative data and median (inter-quartile range; IQR) for quantitative data. Results of cytokine secretion and mRNA expression levels are presented as median and IQR for each group. Comparison of cytokine secretion and mRNA expression median levels between groups was performed through the nonparametric Mann-Whitney test. In addition, the proportion of women with detectable levels of cytokine secretion was compared between groups through the Chi-square test or the Fisher exact test, when necessary. Undetectable cytokine median levels were replaced by half of the value of the detectability threshold. No comparison of cytokine median levels was performed if less than $50 \%$ of women in one group had a detectable level.

Cytokine secretion or mRNA expression levels were compared in HIV-1 infected women according to the type, timing or duration of antiretroviral (ARV) prophylaxis. ZDV duration was divided into 2 categories according to the median, 72 hours. The time interval between NVP intake and delivery was also categorized according to the rounded median, 5 hours. Furthermore, this 5-hour cut-off value is relevant in terms of pharmacokinetics since it has been shown that the peak NVP plasma concentration is reached 3 to 7 hours after single dose intake during labor $[17,20]$. Comparisons were performed through the Mann-Whitney test for quantitative results (levels of secretion or mRNA expression) and through the Chi-square test or the Fisher exact test, when necessary, for qualitative results (proportion of women with detectable secretion levels). Comparisons yielding a $p<0.05$ were considered significant. 


\section{RESULTS}

\section{Characteristics of the Study Population}

The demographic and gestational characteristics of the groups of HIV-1 infected and uninfected pregnant women are shown in Table $\mathbf{1}$. The median age at delivery was not significantly different between the 2 groups (26 versus 28 years, $p=0.56$ ), while the median gestational age at delivery was higher in the group of HIV-1 infected women (39.4 versus 38.6 weeks, $p=0.05$ ). The antiretroviral prophylaxis received and biological characteristics are shown for the group of HIV-1 infected women. Eighteen percent of HIV-1 infected women received ZDV prophylaxis only.

\section{Infant HIV-1 Status}

The HIV-1 status could be determined for 60 of the 61 infants born to HIV-1 infected mothers - one was lost to follow up. Of the 60 infants with known HIV status, none were found to be HIV-1 infected.

\section{No Difference in the Expression Levels of Cytokine Se- cretion Between HIV-1 Infected and Uninfected Women}

Cytokines were quantified in placental culture supernatants of HIV-1 infected women and expression levels were compared to those of uninfected women (Table 2). The proportion of samples with detectable expression levels of IL10 , TNF- $\alpha$, and LIF as well as the median expression levels of LIF, IL-8, IL-15, IL-16, and RANTES were not significantly different between HIV-1 infected and uninfected women (Table 2).

No Difference in the Expression Levels of Cytokine mRNA Between HIV-1 Infected and Uninfected Women

Expression levels of mRNA for the pro-inflammatory cytokines TNF- $\alpha$ and IL-8, the anti-inflammatory cytokine
IL-10, and the chemokine SDF-1 were quantified in placental explants of HIV-1 infected and uninfected women (Table 3). A trend toward lower TNF- $\alpha$ mRNA median expression levels was observed in placentas of HIV-1 infected women $(p=0.06)$ while the median expression levels of SDF-1, IL8 , and IL-10 mRNA of HIV-1 infected women were not significantly different from those of HIV-1 uninfected women.

\section{Placenta Inflammatory Profile in Immunocompromised Women}

Analysis of the relation between the maternal CD4 level and placental cytokine profile showed that women $(\mathrm{n}=19)$ with CD4 count less than or equal to 250 cells $/ \mathrm{mm}^{3}$ expressed higher placental TNF- $\alpha$ mRNA $(2.7$ versus $1.1 \times$ cal, $p=0.007)$ and IL-8 secretion levels $(2,013$ versus 1,517 $\mathrm{pg} / \mathrm{ml}, p=0.02$ ) than women with CD4 count higher than 250 cells $/ \mathrm{mm}^{3}(\mathrm{n}=42)$.

\section{Long Duration of ZDV Prophylaxis is Associated with Higher Placental IL-10 Secretion Level}

We then assessed among HIV-1 infected women the effect of the duration of ZDV prophylaxis on the level of cytokine expression. TNF- $\alpha$ and IL-10 secretions were analyzed since TNF- $\alpha$ is a pro-inflammatory cytokine known to stimulate HIV-1 replication and IL-10 is an anti-inflammatory cytokine which inhibits the expression of TNF- $\alpha$ mRNA. The median expression level of TNF- $\alpha$ mRNA of women who had a long ZDV prophylaxis duration ( $>72$ days) was not significantly different from that of women who had a ZDV prophylaxis duration less than or equal to 72 days $(1.8$ versus $1.7 \times \mathrm{cal}, p=$ $0.30)$. In contrast, the proportion of supernatants with detectable levels of IL-10 was significantly higher in women who received ZDV prophylaxis for more than 72 days than in those who had a ZDV prophylaxis duration less than or equal to 72 days $(32 \%$ versus $7 \%, p=0.01)$ (Fig. 1).

Table 1. Characteristics of the Study Population

\begin{tabular}{|l|c|c|}
\hline \multicolumn{1}{|c|}{ Enrolment Sites } & HIV-1 Infected Women (n= 61) & HIV-1 Uninfected women (n= 38) \\
\hline \hline & & 2 \\
Maharaj Nakorn Chiang Mai Hospital & 49 & 21 \\
Health Promotion Center Region 10 & 7 & 3 \\
Lamphun Hopital & 3 & 2 \\
Nakornping Hospital & & $28(23-30)$ \\
\hline Characteristics: & $26(22-31)$ & $38.6(38.0-39.0)$ \\
\hline Age at delivery (years) & $39.4(37.9-40.4)$ & Not Relevant \\
\hline Gestational age at delivery (weeks) & $29(28-39)$ & Not Relevant \\
\hline Gestational age at ZDV prophylaxis initiation $($ weeks) & $72(54-81)$ & Not Relevant \\
\hline Duration of ZDV prophylaxis (days) & $50(82 \%)$ & Not Relevant \\
\hline NVP plus ZDV prophylaxis & $4.4(1.9-8.2)(\mathrm{n}=46)$ & Not Relevant \\
\hline Time to delivery after NVP dosing (hours) & $332(241-494)$ & Not Relevant \\
\hline CD4 cell count before 28 weeks gestation $\left(\right.$ cells $\left./ \mathrm{mm}^{3}\right)$ & $4.1(3.3-4.6)(\mathrm{n}=57)$ & \\
\hline HIV RNA load at delivery (log 10 copies/ml) & & \\
\hline The data are expressed as median (interquartile range) or $\mathrm{n}$. & & \\
\hline
\end{tabular}

The data are expressed as median (interquartile range) or $n$. 
Table 2. Cytokine Secretion Levels in Supernatants of 24h-Culture of Placental Explants from HIV-1 Infected and Uninfected Pregnant Women

\begin{tabular}{|c|c|c|c|}
\hline Secretion of Cytokines and Chemokines & HIV-1 Infected Women $(n=61)$ & HIV-1 Uninfected Women $(n=38)$ & $p$ \\
\hline IL-10 detectable samples (\%) & 20 & 18 & $0.88^{\mathrm{b}}$ \\
\hline TNF- $\alpha$ detectable samples (\%) & 48 & 32 & $0.12^{\mathrm{b}}$ \\
\hline LIF detectable samples (\%) & 75 & 74 & $0.85^{\mathrm{b}}$ \\
\hline LIF (UI/L) & $55(18-87)$ & $50(17-107)$ & $0.99^{\mathrm{c}}$ \\
\hline IL-8 $(\mathrm{pg} / \mathrm{ml})^{\mathrm{a}}$ & $1787(1086-2271)$ & $1840(1086-2702)$ & $0.81^{\mathrm{c}}$ \\
\hline $\mathrm{IL}-15(\mathrm{pg} / \mathrm{ml})^{\mathrm{a}}$ & $11.0(8.2-16.1)$ & $10.8(6.9-15.7)$ & $0.48^{\mathrm{c}}$ \\
\hline $\mathrm{IL}-16(\mathrm{pg} / \mathrm{ml})^{\mathrm{a}}$ & $404(253-585)$ & $424(249-512)$ & $0.83^{\mathrm{c}}$ \\
\hline RANTES (pg/ml) ${ }^{\mathrm{a}}$ & $506(296-726)$ & $477(359-795)$ & $0.86^{\mathrm{c}}$ \\
\hline
\end{tabular}

The data are expressed as percentage of detectable samples and/or median (interquartile range).

${ }^{a}$ All samples had detectable level of cytokines.

${ }^{6}$ Chi-square test.

${ }^{\mathrm{c}}$ Mann-Whitney test.

Table 3. Placental Cytokine mRNA Levels

\begin{tabular}{|c|c|c|c|}
\hline Expression of Cytokine and Chemokine mRNA $(\times$ cal) & HIV-1 Infected women $(\mathbf{n}=\mathbf{6 1})$ & HIV-1 Uninfected Women $(\mathbf{n}=\mathbf{3 8})$ & $\boldsymbol{p}^{\mathbf{a}}$ \\
\hline \hline SDF-1 & $2.8(1.6-6.7)$ & $3.2(1.9-6.4)$ & 0.95 \\
\hline TNF- $\alpha$ & $1.8(0.8-4.9)$ & $2.8(1.7-4.5)$ & 0.06 \\
\hline IL-8 & $2.4(0.6-6.1)$ & $3.1(1.0-7.4)$ & 0.37 \\
\hline IL-10 & $2.5(1.2-6.2)$ & $3.2(1.8-5.3)$ & 0.36 \\
\hline
\end{tabular}

The data are expressed as median (interquartile range). The cytokine mRNA levels are expressed as multiples of the results obtained with a reference cDNA prepared from a term placental tissue of a HIV-1 uninfected woman and used as a calibrator $(\times$ cal $)$.

${ }^{\mathrm{a}}$ Mann-Whitney test.

\section{Administration of NVP Less Than 5 Hours Prior to De- livery is Associated with Higher Placental IL-10 Secre- tion Level}

The median expression level of TNF- $\alpha$ mRNA in women who received ZDV plus NVP was comparable to that of women who received ZDV only (1.6 versus $2.3 \times$ cal, $p=$ $0.24)$. Among women who received a single dose of NVP during labor, TNF- $\alpha$ mRNA expression levels were similar in those who received NVP at least 5 hours prior to delivery and those who received NVP less than 5 hours prior to delivery (1.1 versus $1.6 \times \mathrm{cal}, p=0.19)$. Similarly, the proportion of placentas with detectable expression levels of IL-10 was not different between women who received ZDV plus NVP and those who received ZDV only (18\% versus $27 \%, p=$ 0.48) (Fig. 2A). However, among women who received a single dose of NVP during labor, detectable IL-10 secretion levels were found in higher proportion of those who received NVP less than 5 hours prior to delivery than in those who received NVP later (32\% versus, $5 \%, p=0.02)$ (Fig. 2B).

\section{DISCUSSION}

We report here, for the first time, an association between a higher expression level of the anti-inflammatory IL-10 cytokine in the placenta and either a longer ZDV prophylaxis duration or a shorter time to delivery after intake of NVP during labor.

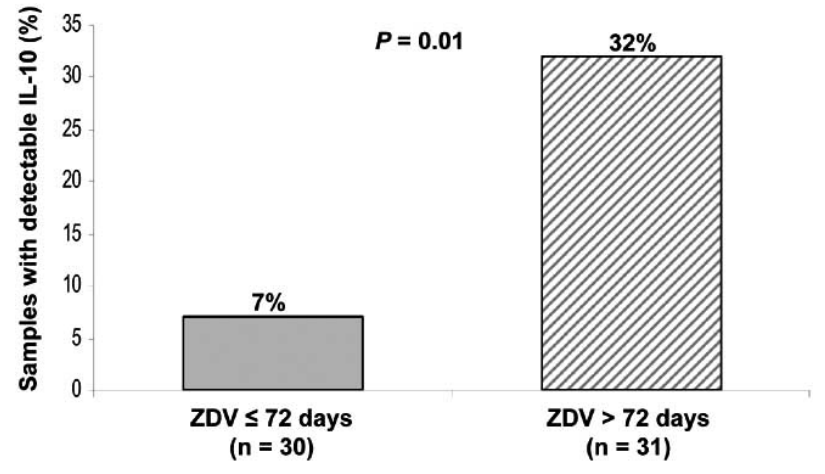

Fig. (1). Proportion of detectable IL-10 levels $(\geq 5 \mathrm{pg} / \mathrm{ml})$ in placental culture supernatants of HIV-1 infected women according to ZDV prophylaxis duration (72 days). IL-10 was evaluated by ELISA.

HIV-1 infected patients usually exhibit high levels of inflammatory cytokines. The fact that no significant differences were observed between the median placental cytokine levels of HIV-1 infected and uninfected women suggests a possible effect of ARV in regulating cytokine expression at the placental level. However, this hypothesis could not be ascertained since all HIV-1 infected women in the study received ZDV or ZDV plus NVP. In the present study, placentas of HIV-1 infected women showed a trend toward lower median expression of TNF- $\alpha$ mRNA than placentas 


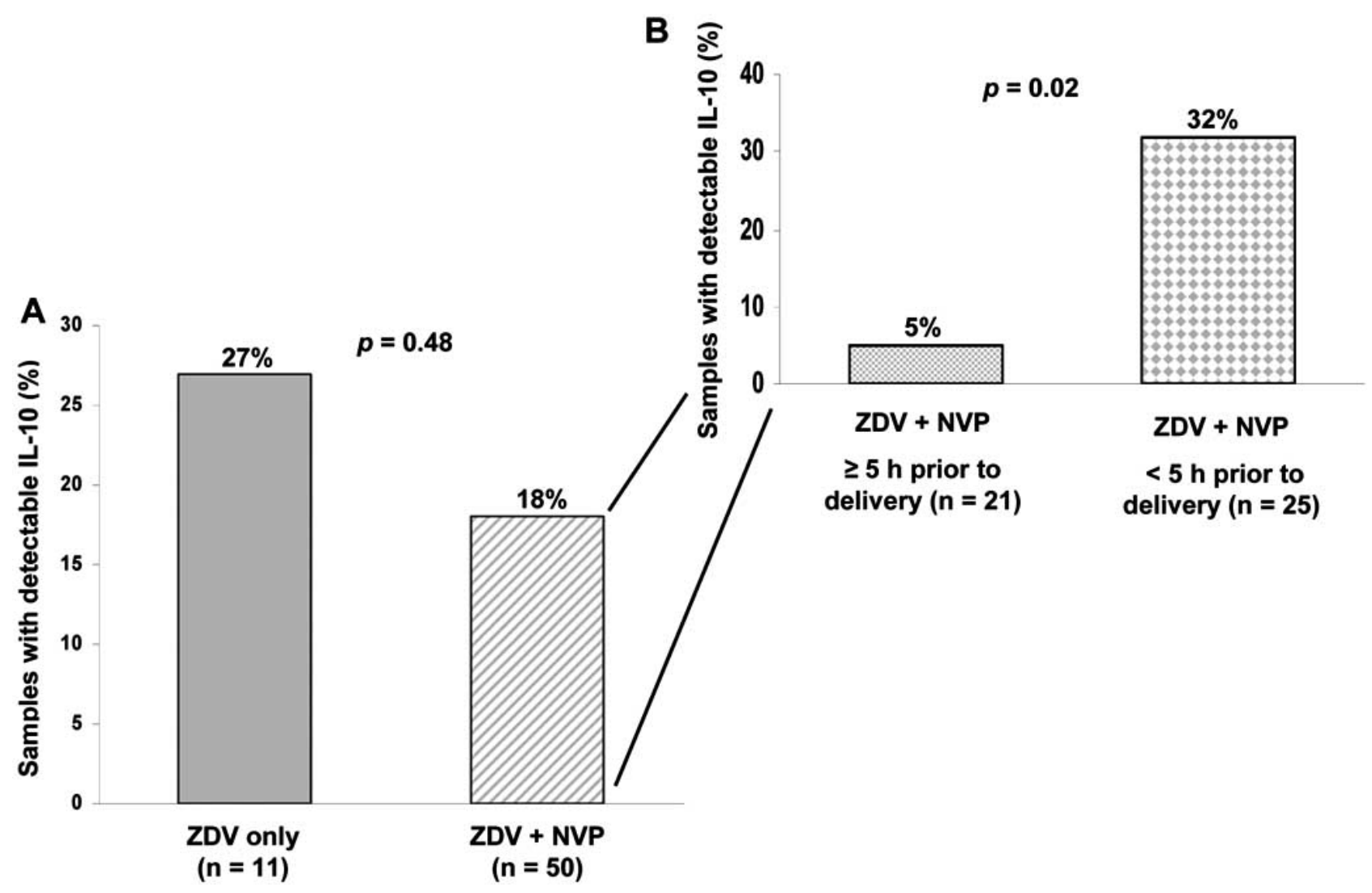

Fig. (2). Proportion of detectable IL-10 levels ( $\geq 5 \mathrm{pg} / \mathrm{ml})$ in placental culture supernatants of HIV-1 infected women according to the ARV prophylaxis regimen. (A) Women received ZDV only or ZDV plus a single dose of NVP at onset of labor. (B) Women received NVP less than 5 hours or at least 5 hours prior to delivery. IL-10 was evaluated by ELISA. Time from NVP intake to delivery was not available for 4 HIV-1 infected women.

of HIV-1 uninfected women (Table 3), suggesting that ZDV or ZDV plus NVP might decrease the expression of TNF- $\alpha$ mRNA. This hypothesis is consistent with our previous in vitro study showing a decrease of TNF- $\alpha$ transcripts when ZDV was added to normal placental histoculture [21]. Thus, ZDV or the combination of ZDV plus NVP may contribute to the normalization of the placental cytokine balance in HIV-1 infected women. This is also consistent with the Hygino et al. report [22] showing similar lymphoproliferation and IL-4, IL-10, IFN- $\gamma$ and TNF- $\alpha$ levels in cord blood of neonates born to HIV-1 infected mothers who controlled their plasma HIV RNA and neonates born to HIV-1 uninfected mothers. Moreover, neonates born to HIV-1 infected mothers who received ZDV plus NVP during pregnancy had significantly higher IL-10 levels in their plasma than those born to untreated HIV-1 infected mothers.

In our study, a long ZDV prophylaxis duration $(>72$ days) was associated with a higher proportion of detectable IL-10 secretion levels $(p=0.01)$. This proportion was also higher when NVP was given less than 5 hours prior to delivery $(p=0.02)$. These data suggest that ZDV itself could increase the production of IL-10 and this effect might be synergized by NVP. The effect of NVP on IL-10 secretion, which cannot be observed more than 5 hours after administration, is more difficult to interpret and could be related to a short duration of labor. Another possible explanation is that initial induction of IL-10 secretion during the first 5 hours after NVP intake could be followed by inactivation of IL-10 secretion through a negative feedback mechanism as a result of prolonged exposure to NVP [23-25]. However, this "anergic" state was not described by Schramm et al., who reported an increase in plasma activation markers such as neopterin, $\beta_{2}$-microblobulin, and soluble L-selectin in women exposed to SD-NVP [22]. Another hypothesis could be that the secreted IL-10 binds to its receptors and can no longer be detected in our system 5 hours after NVP intake. A recent paper also highlighted the impact of the duration of ARV treatment on the risk of HIV-1 MTCT in women with a low viral load and vaginal delivery [26].

Although an alteration of the TNF- $\alpha /$ IL-10 balance toward a pro-inflammatory cytokine profile has been associated with HIV-1 disease progression [27, 28], whether or not the increase of anti-inflammatory IL-10 cytokine, resulting from ZDV or ZDV plus NVP, may participate in the control of HIV-1 replication is still unclear. The fact that immunocompromised women expressed higher placental TNF- $\alpha$ mRNA and IL-8 secretion levels likely reflects their more advanced disease stage. The sample size was too limited to analyze these results according to the type and duration of the ARV prophylaxis. In comparing placental cytokine profiles from transmitting versus non-transmitting mothers, Behbahani et al. reported an imbalance of placental cytokine 
distribution towards lower levels of IL-2 and higher levels of IL-4 and IL-10 cytokines in the placenta of HIV-1 non transmitting mothers [29]. In the present study, it was not possible to assess whether or not placental cytokine levels were associated with perinatal HIV-1 transmission since no infant was infected by HIV-1.

The exact molecular mechanisms by which ZDV and NVP control HIV-1 transmission through placental cytokine balance are not known. A possible mechanism could be that ZDV and NVP exert their activity through the transcriptional NF-kB pathway to down-regulate TNF- $\alpha$ gene expression, thus diminishing HIV replication [30]

In conclusion, this study demonstrates that longer zidovudine prophylaxis and shorter time to delivery after NVP dosing are associated with a higher placental antiinflammatory IL-10 expression. This effect may contribute to reducing MTCT of HIV-1 through a reduction in HIV replication at the placental level. Therefore, the effect of ARV drugs on placental cytokine profiles should be considered in developing further strategies to prevent HIV-1 MTCT.

\section{ACKNOWLEDGMENTS}

The authors wish to thank all the mothers who participated in this study, Dr. Jean-Luc Taupin for the LIF dosage and Mrs. Jacqueline Regnault for technical assistance. We also wish to thank the PHPT staff for their support and commitment, Prof. Kenneth Mac Intosh for his critical review of the manuscript, and Intira Collins and Catherine Kress for their help in editing this manuscript.

This work was supported by grants from the Agence Nationale de Recherches sur le Sida et les hepatites virales (ANRS \#1267, Paris France), INSERM, Institut Pasteur, and the National Institutes of Health, USA (R01 HD 39615).

Sakorn Pornprasert received a scholarship from the Thai Staff Development Project of the Ministry of University Affairs in Thailand. Albert Faye was supported by a fellowship award from Ensemble contre le SIDA (Sidaction).

\section{ANRS 1267 Study Group and HIV-1 PMTCT-PlaNet}

Ahidjo Ayouba (Institut Pasteur, Paris, France), Françoise Barré-Sinoussi (Institut Pasteur, Paris, France), Sanupong Chailert (IRD URI 174/PHPT, Chiang Mai Thailand), Gérard Chaouat (INSERMU131, Clamart, France), Muriel Derrien (ICGM, Paris, France), Guillermina Dolcini (Facultad de Medicina, Universidad de Buenos Aires, Argentine), Nicole Eteki (Maternité Principale, Hôpital Central de Yaoundé, Cameroon), Albert Faye (Institut Pasteur, Paris, France et INSERMU131, Clamart, France), Vorapin Gomuthbutra (Nakornping Hospital, Chiang Mai, Thailand), Anfumbom Jude Kfutwah (Centre Pasteur du Cameroun, Yaoundé, Cameroon), Odette Kouo (Centre de Santé Mgr J. ZOA de NKOLNDONGO, Yaoundé, Cameroon), Marc Lallemant (IRD URI 174/PHPT, Chiang Mai, Thailand), Pranee Leechanachai (Faculty of Associated Medical Sciences, CMU, Chiang Mai, Thailand), Sophie Le Coeur (Institut National d'Etudes Démographiques, Paris, France), Brigitte Lemen (Centre de Santé Mgr J. ZOA de NKOLNDONGO, Yaoundé, Cameroon), Aram Limtrakul (Health Promotion Center Region 10 Hospital, Chiang Mai,
Thailand), Juan Maldonado-Estrada (Facultad de Ciencas Agrarias, Universidad de Antioquia, Medellin, Colombia), Jean-Yves Mary (INSERM U717, Paris, France), Wanmanee Matanasaravoot (Lamphun Hospital, Lamphun, Thailand), Elisabeth Menu (Institut Pasteur, Paris, France), Eric Nerrienet (Institut Pasteur du Cambodge, Phnom Penh, Cambodia), Nicole Ngo-Giang-Huong (HSPH/IRD URI 174, Chiang Mai, Thailand), Bernadette Njinku (Centre Pasteur du Cameroun, Yaoundé, Cameroon), Sakorn Pornprasert (Faculty of Associated Medical Sciences, CMU, Chiang Mai, Thailand), Sungwal Rugpao (Maharaj Nakorn Chiang Mai Hospital, Thailand), Gabriella Scarlatti (DIBIT, San Raffaele Scientific Institute, Milano, Italy), Pannee Sirivatanapa (Suan Dok Hospital, Chiang Mai, Thailand), Mathurin Tejiokem (Centre Pasteur du Cameroun, Yaoundé, Cameroon), Gilbert Téné (Centre Mère et Enfant, Fondation C. BIYA, Yaoundé, Cameroon).

\section{REFERENCES}

[1] Douglas GC, King BF. Maternal-fetal transmission of human immunodeficiency virus: a review of possible routes and cellular mechanisms of infection. Clin Infect Dis 1992; 15: 678-91.

[2] Zachar V, Norskov-Lauritsen N, Juhl C, Spire B, Chermann JC Ebbesen P. Susceptibility of cultured human trophoblasts to infection with human immunodeficiency virus type 1. J Gen Virol 1991; 72: $1253-60$

[3] Zachar V, Spire B, Hirsch I, Chermann JC, Ebbesen P. Human transformed trophoblast-derived cells lacking CD4 receptor exhibit restricted permissiveness for human immunodeficiency virus type 1. J Virol 1991; 65: 2102-7.

[4] Dolcini G, Derrien M, Chaouat G, Barre-Sinoussi F, Menu E. Cellfree HIV type 1 infection is restricted in the human trophoblas choriocarcinoma BeWo cell line, even with expression of CD4, CXCR4 and CCR5. AIDS Res Hum Retroviruses 2003; 19: 85764.

[5] Brabin BJ, Romagosa C, Abdelgalil S, et al. The sick placenta-the role of malaria. Placenta 2004; 25: 359-78.

[6] Rogerson SJ, Brown HC, Pollina E, et al. Placental tumor necrosis factor alpha but not gamma interferon is associated with placental malaria and low birth weight in Malawian women. Infect Immun 2003; 71: 267-70.

[7] Fievet N, Moussa M, Tami G, et al. Plasmodium falciparum induces a Th1/Th2 disequilibrium, favoring the Th1-type pathway, in the human placenta. J Infect Dis 2001; 183: 1530-4.

[8] Reuben JM, Gonik B, Li S, Loo L, Turpin J. Induction of cytokines in normal placental cells by the human immunodeficiency virus. Lymphokine Cytokine Res 1991; 10: 195-9.

[9] Reuben JM, Turpin JA, Lee BN, et al. Induction of inflammatory cytokines in placental monocytes of gravidae infected with the human immunodeficiency virus type 1 . J Interferon Cytokine Res 1996; 16: 963-71.

[10] Gallagher M, Malhotra I, Mungai PL, et al. The effects of maternal helminth and malaria infections on mother-to-child HIV transmission. Aids 2005; 19: 1849-55.

[11] Vidricaire G, Tardif MR, Tremblay MJ. The low viral production in trophoblastic cells is due to a high endocytic internalization of the human immunodeficiency virus type 1 and can be overcome by the pro-inflammatory cytokines tumor necrosis factor-alpha and interleukin-1. J Biol Chem 2003; 278: 15832-41.

[12] Zachar V, Fink T, Koppelhus U, Ebbesen P. Role of placental cytokines in transcriptional modulation of HIV type 1 in the isolated villous trophoblast. AIDS Res Hum Retroviruses 2002; 18 839-47.

[13] Connor EM, Sperling RS, Gelber R, et al. Reduction of maternalinfant transmission of human immunodeficiency virus type 1 with zidovudine treatment. Pediatric AIDS Clinical Trials Group Protocol 076 Study Group. N Engl J Med 1994; 331: 1173-80.

[14] Guay LA, Musoke P, Fleming T, et al. Intrapartum and neonatal single-dose nevirapine compared with zidovudine for prevention of mother-to-child transmission of HIV-1 in Kampala, Uganda: HIVNET 012 randomised trial. Lancet 1999; 354: 795-802. 
[15] Lallemant M, Jourdain G, Le Coeur S, et al. Single-dose perinatal nevirapine plus standard zidovudine to prevent mother-to-child transmission of HIV-1 in Thailand. N Engl J Med 2004; 351: 217 28.

[16] Sperling RS, Shapiro DE, Coombs RW, et al. Maternal viral load, zidovudine treatment, and the risk of transmission of human immunodeficiency virus type 1 from mother to infant. Pediatric AIDS Clinical Trials Group Protocol 076 Study Group. N Engl J Med 1996; 335: 1621-9.

[17] Musoke P, Guay LA, Bagenda D, et al. A phase I/II study of the safety and pharmacokinetics of nevirapine in HIV-1-infected pregnant Ugandan women and their neonates (HIVNET 006). Aids 1999; 13: 479-86.

[18] Faye A, Pornprasert S, Mary JY, et al. Characterization of the main placental cytokine profiles from HIV-1-infected pregnant women treated with anti-retroviral drugs in France. Clin Exp Immunol 2007; 149: 430-9.

[19] Taupin JL, Gualde N, Moreau JF. A monoclonal antibody based elisa for quantitation of human leukaemia inhibitory factor. Cytokine 1997; 9: 112-8.

[20] Mirochnick M, Fenton T, Gagnier P, et al. Pharmacokinetics of nevirapine in human immunodeficiency virus type 1-infected pregnant women and their neonates. Pediatric AIDS Clinical Trials Group Protocol 250 Team. J Infect Dis 1998; 178: 368-74.

[21] Pornprasert S, Faye A, Mary JY, et al. Down modulation of TNFalpha mRNA placental expression by AZT used for the prevention of HIV-1 mother-to-child transmission. Placenta 2006; 27: 989-95.

[22] Schramm DB, Kuhn L, Gray GE, Tiemessen CT. In vivo effects of HIV-1 exposure in the presence and absence of single-dose nevirapine on cellular plasma activation markers of infants born to
HIV-1-seropositive mothers. J Acquir Immune Defic Syndr 2006; 42: 545-53.

[23] de Waal Malefyt R, Abrams J, Bennett B, Figdor CG, de Vries JE. Interleukin 10(IL-10) inhibits cytokine synthesis by human monocytes: an autoregulatory role of IL-10 produced by monocytes. J Exp Med 1991; 174: 1209-20.

[24] Knolle PA, Uhrig A, Protzer U, et al. Interleukin-10 expression is autoregulated at the transcriptional level in human and murine Kupffer cells. Hepatology 1998; 27: 93-9.

[25] Roth I, Corry DB, Locksley RM, Abrams JS, Litton MJ, Fisher SJ. Human placental cytotrophoblasts produce the immunosuppressive cytokine interleukin 10. J Exp Med 1996; 184: 539-48.

[26] Warszawski J, Tubiana R, Le Chenadec J, et al. Mother-to-child HIV transmission despite antiretroviral therapy in the ANRS French Perinatal Cohort. Aids 2008; 22: 289-99.

[27] Than $\mathrm{S}, \mathrm{Hu} \mathrm{R}$, Oyaizu $\mathrm{N}$, et al. Cytokine pattern in relation to disease progression in human immunodeficiency virus-infected children. J Infect Dis 1997; 175: 47-56.

[28] Breen EC. Pro- and anti-inflammatory cytokines in human immunodeficiency virus infection and acquired immunodeficiency syndrome. Pharmacol Ther 2002; 95: 295-304.

[29] Behbahani H, Popek E, Garcia P, et al. Up-regulation of CCR5 expression in the placenta is associated with human immunodeficiency virus-1 vertical transmission. Am J Pathol 2000; 157: 18118 .

[30] Ghosh SK, Wood C, Boise LH, et al. Potentiation of TRAILinduced apoptosis in primary effusion lymphoma through azidothymidine-mediated inhibition of NF-kappa B. Blood 2003; 101: 2321-7. 